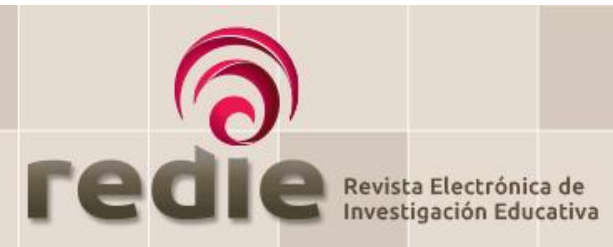

ISSN: $1607-4041$

http://redie.uabc.mx

Vol. 19, Núm. 3, 2017

\title{
Consejos Técnicos Escolares, un espacio de colaboración para evaluar el ejercicio docente en Educación Básica
}

\author{
Technical School Boards: A Space for Collaboration in Assessing \\ Teaching Practice in Basic Education
}

Rosa María González Isasi (1) rosygisa@hotmail.com

Carlos Humberto de la Garza Saldívar (1) carhum0064@hotmail.com

Martha Elvira de León Hernández (2) marthadeleonrod@hotmail.com

(1) Universidad Pedagógica Nacional

(2) Secretaría de Educación Tamaulipas

(Recibido: 23 de febrero de 2016; Aceptado para su publicación: 26 de abril de 2016)

Cómo citar: González, R. M., De la Garza, C. H. y De León, M. E. (2017). Consejos Técnicos Escolares, un espacio de colaboración para evaluar el ejercicio docente en Educación Básica. Revista Electrónica de Investigación Educativa, 19(3), 24-32.

https://doi.org/10.24320/redie.2017.19.3.1272

\section{Resumen}

La presente investigación (estudio de caso) se desarrolló en dos escuelas de Educación Básica. En ella participaron 54 educadores: 45 de educación primaria y 9 de preescolar. El propósito fue estudiar cómo la Comunidad de Práctica (CoP) implementada en las reuniones de Consejo Técnico Escolar (CTE) propiciaría el análisis de las prácticas de evaluación de aprendizajes a partir del trabajo colaborativo. En cada escuela se desarrollaron cuatro reuniones de aproximadamente dos horas que fueron registradas en video. Se encontró que los profesores identificaron áreas de oportunidad para mejorar la práctica mediante el trabajo colaborativo, fortaleciendo una relación de apertura entre ellos y reflexionando sobre sus prácticas evaluativas.

Palabras clave: Comunidad de práctica, Consejo Técnico Escolar, trabajo colaborativo, evaluación docente.

\section{Abstract}

This research - a case study - was conducted in two schools at the basic education stage (educación básica) and 54 educators - 45 from primary and 9 from preschool education - participated. The purpose was to study how the Practice Community (PC) introduced in Technical School Board (TSB) meetings would benefit the analysis of learning assessment practices from collaborative work. Each school held four video-recorded meetings of approximately two hours in duration. It was found that teachers identified opportunity areas for improving practice through collaborative work, which led to a stronger, more open relationship between them and reflection on their assessment practices. 


\section{Introducción}

La evaluación de la práctica docente es una tarea que desde hace tiempo ha estado planteada como necesaria, pues permite identificar áreas de oportunidad para mejorar las prácticas de los profesores, y atender esos aspectos con procesos de profesionalización. Una de las formas de atender esa acción es, como propuso Casanova (2004), la autoevaluación por parte de los profesores, pues:

[Es] un tipo de evaluación que toda persona realiza de forma permanente a lo largo de su vida, ya que continuamente toman decisiones en función de la valoración positiva o negativa de una actuación específica, una relación tenida, un trabajo llevado a cabo, etc. (p. 31).

Pero las autoevaluaciones pueden dejar de lado uno o más aspectos de la práctica docente que el profesor no puede visualizar. Por ello se hace necesario que ese proceso se realice con la participación del colectivo docente. Esto puede hacerse a través de análisis colaborativo para detectar las áreas de oportunidad a través del diálogo, la reflexión, el análisis y la interacción. Para desarrollar ese diálogo, en educación básica pueden aprovecharse los espacios de las reuniones colegiadas del Consejo Técnico Escolar (CTE), como un espacio en el que, por reglamento, se deben analizar temáticas relativas al plan y programas de estudio, métodos de enseñanza y evaluación. Es por ello que son considerados como espacios en los que los profesores tienen la oportunidad de compartir experiencias, movilizar sus saberes y reforzar áreas de oportunidad. Este espacio se conforma institucionalmente por el director y los profesores, de quienes se requiere decisión e involucramiento directo, con el propósito de analizar y hacer recomendaciones sobre diversos temas escolares, entre los cuales está la capacitación del personal docente (Secretaría de Educación Pública, 1982).

Para el cumplimiento de la norma que regula el funcionamiento de los CTE se establece que se realicen desde el inicio del año escolar reuniones colegiadas mensuales como espacios para el intercambio de ideas y la toma de decisiones respecto a la tarea pedagógica, de gestión y administrativa de la escuela. Orler y Abendaño $(2009$, p. 1) identificaron a esas reuniones como "un espacio en el cual los docentes aportan diferentes miradas sobre los alumnos y la marca de los procesos de enseñanza y aprendizaje. En estos espacios el profesor o tutor juega un rol particular".

Una de las tareas que deben realizarse en los CTE es la discusión y elaboración del Plan Anual de Trabajo (PAT), en donde el Asesor Técnico Pedagógico (ATP) apoya en el abordaje de las temáticas y los docentes realizan diferentes producciones (Secretaría de Educación Pública, [SEP] 1982); todo ello orientado a: a) el beneficio del proceso enseñanza-aprendizaje y de sus alumnos, b) la relación con los padres de familia y la comunidad en la que se encuentra inmersa la institución y c) la gestión organizativa.

Institucionalmente se contempla que el espacio de los CTE permitirá que el profesor se encuentre actualizado. Sin embargo, como señala Ezpeleta (1990), son muy pocas las escuelas en donde se reflexiona sobre los asuntos pedagógicos, en unos casos "ocupan un lugar periférico con relación a otras instancias de la organización institucional, especialmente las Comisiones" (p. 17).

Fierro y Rojo (1994) señalan que los CTE son utilizados para muchas cosas antes de ocuparse de lo pedagógico, lo que demuestra el lugar secundario que tiene el análisis de la práctica; frecuentemente están supeditados a las cuestiones administrativas, como lo planteó Ezpeleta (1990). De allí que la razón por la que algunos profesores sólo acudan a las reuniones de CTE para acatar una disposición institucional.

Por otro lado, el CTE se constituye en un espacio para socializar diversas concepciones, "costumbres, hábitos, tradiciones y mitos que permiten, o no, desarrollar una cultura académica" (López, 2005, p. 324), pero en el que se hacen presentes una serie de rituales de la práctica educativa. Esos rituales frecuentemente son opuestos a las nuevas tendencias planteadas por la SEP, quien ha hecho énfasis en las capacitaciones a través de cursos o talleres presenciales de corta duración desarrollados en cascada, que son definidos desde las necesidades de las instituciones organizadoras, generalmente para capacitar a los profesores en la implementación de nuevos programas o reformas educativas sin considerar las características de los participantes, por lo que tienen poco impacto en la formación de los profesores (Messina, 2002). 
De allí que los CTE pueden constituirse en un espacio para analizar, entre otros aspectos de la función de la escuela, la evaluación de la práctica docente de los profesores en trabajo colaborativo. Esta evaluación, a su vez, puede detonar la transformación del quehacer de los profesores, ya que con su proceso y resultados se pueden movilizar saberes al compartir experiencias, puntos de vista, concepciones relacionadas sobre ese quehacer y no sólo discutir elementos teóricos, de política educativa o de administración escolar, como comúnmente se realiza en las escuelas. Al realizar la evaluación de la práctica docente en colectivo se cubren aspectos que el profesor aislado pasa por alto.

Así, se pueden desarrollar procesos de autoevaluación, acompañados y contrastados con co-evaluaciones realizadas por pares y hetero-evaluaciones realizadas por las autoridades educativas de la escuela, como el director, ATP o supervisor escolar. Esto resulta relevante porque, como señalaron Moliner, Castellón y Loren (2010), en algunos países europeos y latinoamericanos se ve la capacitación y la formación continua como un proceso de acompañamiento del profesor, invitándolo a reflexionar y analizar su trabajo diario en un contexto real, permitiéndole la búsqueda de nuevas formas de actuar en su salón de clase. La profesionalización docente debe ser un ejercicio, a la par del trabajo en la institución, en el que los profesores sean los protagonistas y su acción se centre en el trabajo colaborativo a través del continuo cuestionamiento y reflexión, que le permita aterrizar en el aula lo compartido en el colectivo. Esto lo logrará al participar activamente en equipos de trabajo, con respeto y tolerancia, solidaridad, en beneficio de la Institución (Álvarez y Romero, 2007).

Bajo esas premisas se desarrolló un estudio cualitativo con el método de estudio de caso en dos escuelas urbanas de Educación Básica: una de educación preescolar y una de educación primaria, en el período semestral de enero a julio de 2015 . El propósito fue propiciar que los participantes analizaran y evaluaran su práctica docente y se plantearan acciones de mejora.

En esas escuelas, los CTE han tenido poco impacto en la conformación de una cultura de la evaluación realizada en forma colaborativa. Aunque los profesores de las dos escuelas estudiadas habían participado en diversas reuniones de CTE, poco o nulo tiempo se había dedicado para el análisis y evaluación de la práctica docente. Así, se consideró que desarrollar acciones de reflexión y evaluación sobre las prácticas cotidianas que los profesores realizaban en el aula podía apoyarlos a reconocer los aspectos que pudieran ser objeto de mejora y desarrollar acciones que pudieran aplicarse en el aula.

Para el desarrollo de la investigación se plantearon las preguntas: ¿Cómo las evaluaciones de la práctica docente realizadas en forma colaborativa en el CTE aportan elementos a la conformación de una cultura de evaluación de la práctica docente que propicie el desarrollo de propuestas de mejora?, y ¿cómo la participación en trabajo colaborativo desarrollado en las reuniones de CTE impacta en un reconocimiento de ellas como un espacio para la formación continua?

\section{Método}

En el proceso hubo un coordinador y dos monitores: uno en cada una de las escuelas, en las que tenían una relación laboral. El proceso se desarrolló en dos fases: en la primera se analizó la situación que privaba en el desarrollo de las reuniones de CTE y se invitó al personal a participar en la CoP (Comunidad de Práctica) de su escuela. En la segunda etapa los participantes evaluaron colaborativamente clases videograbadas de sus compañeros, quienes aceptaron voluntariamente que se les grabara duante una clase y ellos se autoevaluaron también. Para la evaluación de la práctica docente se realizó el registro de observación videograbado de 6 clases, 3 en cada escuela. Los videos los revisaron considerando estándares de evaluación en forma colaborativa. Una vez evaluadas las clases se realizaron propuestas de mejora a las que los integrantes de la CoP dieron seguimiento. También se entrevistó a algunos profesores después de su participación en las reuniones para conocer su opinión respecto al impacto que el trabajo colaborativo tuvo en su formación y su práctica docente. 


\subsection{Procedimiento}

Aspectos relevantes de la escuela de Educación Primaria. En la dinámica seguida en las reuniones de CTE se realizaba un consenso tras revisar cada exposición de los equipos de profesores, según la temática que se les indicaba en la agenda de trabajo de la reunión, con el objetivo de conocer, discutir o dar respuesta a las necesidades que se habían identificado a nivel de institución, o en forma específica a cada equipo de trabajo. Ese trabajo generalmente reflejaba una participación cooperativa no colaborativa, es decir, cada uno hacía su trabajo y después lo conjuntaban para tener un producto final (Lucero, 2009).

Las reuniones también eran aprovechadas para comentar algunas experiencias de aprendizaje de los alumnos y de manejo de contenidos, tanto las que consideraban positivas como las que no lo fueron. Lo anterior se relaciona con la recomendación del Acuerdo 592 (SEP y Sindicato Nacional de Trabajadores de la Educación, 2011a), en el que se establece, para el trabajo en las escuelas, el desarrollo de "acciones para el descubrimiento, la búsqueda de soluciones, coincidencias y diferencias, con el propósito de construir aprendizajes en colectivo" (p. 21), específicamente en lo relacionado a la construcción de aprendizajes por el colectivo de profesores. Sin embargo, se presentaban dos situaciones críticas: 1) generalmente se analizaban con mínima profundidad las situaciones presentadas y 2 ) en pocas ocasiones se daba seguimiento a los acuerdos tomados en dichas reuniones.

Por otro lado, en la agenda se incluían las temáticas establecidas por el jefe de sector educativo y se adecuaban (según su percepción) a las necesidades de la institución, siempre acordes con el desarrollo del Plan de Estudios y al Plan Anual de Trabajo (PAT). Además, esta tarea la desarrollaban los ATP, quienes en algunos casos no se encontraban debidamente capacitados para desempeñar funciones académicas porque eran profesores de grupo habilitados para desempeñar esa función. Todo ello propiciaba que poco se lograra la generación de un ambiente organizacional estructurado con valores y creencias que contribuyeran a la coherencia de una cultura de la evaluación. Con ello queda claro que los espacios de reuniones colegiadas de CTE poco habían estado siendo utilizadas de forma que apoyaran dinámicamente la mejora de las prácticas evaluativas del quehacer docente. Esto es, no se aprovechaba un espacio que, como señaló Jiménez (2009), constituye una de las formas más dinámicas de aprender sobre la práctica docente y transformarla.

Aspectos relevantes de la escuela de Educación Preescolar. En esta escuela los profesores contaban con pocos momentos para compartir experiencias y diálogos, pues sus jornadas laborales se extendían en tres turnos: el servicio regular, el servicio mixto y el servicio vespertino. Sin embargo, contaban con el espacio de las reuniones mensuales del CTE, para los cuales el reglamento marca que se conforme en todas las escuelas con más de cuatro maestros (Ezpeleta, 1990).

Dentro de esas reuniones mensuales se daba relevancia a las prácticas de evaluación de los aprendizajes; sin embargo, el enfoque en que se orientaban las dinámicas de esas discusiones, no correspondía a un proceso basado en el trabajo colaborativo, sino que se seguía trabajando de forma conductista, directiva, que inhibe la reflexión, ya que se realizaban lecturas en carrusel, esto es, una educadora leía, enseguida otra y así sucesivamente. Después de la lectura se contestaban preguntas sobre al contenido leído. Aunque las respuestas en ocasiones incluían experiencias de quien aportaba, eran pocas y se acomodaban actividades de corte administrativo dejando de lado el proceso de análisis, comprensión, reflexión y trabajo entre pares.

En ese sentido, no resulta difícil comprender que la mayor parte de lo abordado en las reuniones quedara en el discurso. Esto se veía reflejado en las incongruencias en cuanto a la evaluación, porque aunque el manejo del discurso era adecuado, no se discutía la forma de llevarlo a la práctica ni se establecía el compromiso de implementar estrategias pertinentes al nuevo enfoque de evaluación por competencias, por lo que se utilizaban los instrumentos de enfoques anteriores. Por ello era necesario que los profesores fortalecieran la observación de su clase y mejoraran o ampliaran el uso de instrumentos pertinentes para evaluar, señalados en el Plan de Estudios 2011 (SEP, 2011b), porque al no aplicar los fundamentos de estos instrumentos se limita la obtención de evidencias en el proceso de evaluación de los alumnos; como señalaron Marín, Guzmán y Castro (2012), entre los principales problemas que enfrentan los profesores en relación con la evaluación, es que el logro de competencias es aún incipiente; 
en este caso, los profesores no aplicaban instrumentos adecuados para realizar la evaluación.

En ese sentido, era necesario propiciar que los profesores de ambas escuelas reflexionaran sobre su práctica docente. Se consideró analizar la forma pertinente de trabajar en forma dinámica para fortalecer el trabajo de la evaluación de la práctica docente, a través de la CoP en la cual aprovecharan los espacios de las reuniones colegiadas. Al respecto, González, Zamarripa, Cardoso, Martínez y Sánchez (2009, p. 49) señalaron: "Una comunidad de práctica es un espacio informal y temporal cuyo fin es plantear el crecimiento de sus miembros y solventar problemas comunes mediante el establecimiento de un repertorio de conocimientos, producto de su participación".

La participación en una CoP es más o menos comprometida, pero las tareas se comparten, se plantean puntos de vista relevantes de lo que acontece en su cotidianeidad escolar (Wenger, 2002). Al respecto, Scagnoli $(2005$, p. 3) refirió que "el objetivo del trabajo colaborativo es inducir a los participantes a la construcción del conocimiento mediante la exploración, discusión negociación y debate". Esto, como señaló Wenger (2002), se puede lograr en una CoP.

Desarrollo del trabajo colaborativo en CoP dentro del CTE en escuela de Educación Primaria. En el desarrollo de las actividades, lo primero que se hizo fue clarificar lo que era una CoP, ya que no todos estaban familiarizados con la terminología. Desde la fase de sensibilización hubo disposición para conformar la CoP. Respecto a este trabajo colaborativo se identificaron dos aspectos: su efecto en la cultura de la evaluación y la formación continua.

Trabajo colaborativo en la conformación de una cultura de evaluación de la práctica docente. Desde el primer momento los profesores tomaron acuerdos sobre lo que se necesitaba para el trabajo de la CoP. Se plantearon el compromiso de intercambiar experiencias en un marco de respeto y con una continua reflexión. El compromiso se cumplió, pudieron expresar y sugerir ideas y compromisos para el Plan de Mejora, visto como resultado del análisis y evaluación de la práctica docente realizada. Lo anterior era importante, pues trabajar colaborativamente en una CoP no es fácil. Al respecto, González et al. (2013) señalaron:

Trabajar colaborativamente no resulta sencillo, sobre todo cuando lo que se analiza es la propia práctica docente, ya que allí los participantes se ven expuestos a los ojos de sus compañeros y les cuesta mucho trabajo aceptar las críticas que sobre todos sus actos se expresan. Ello aunado a la dificultad de los sujetos de percibirse a sí mismos (p.105).

En todas las actividades planeadas los profesores respetaron los diferentes puntos de vista, reflexionaron sobre lo que se estaba diciendo, reconocieron aciertos y áreas de oportunidad. Esto es, se evidenciaron prácticas reflexivas en colaboración con otros participantes, demostrando lo que Tallaferro (2006) señaló al referirse a la práctica, que "trae consigo mucho más que actos observables, es parte de un sistema de ideas y conocimientos al involucrar valores, actitudes, saberes, formas de ser, pensar, hablar y sentir" ( $p$. 3).

Después de evaluar las clases videograbadas y su experiencia personal y profesional, los profesores tomaron acuerdos sobre los aspectos en que debían poner más énfasis para transformar su práctica docente, también acordaron que en el plan de mejora se tomaran en cuenta las necesidades de su grupo con los estándares de desempeño docente como guías ya que, como expresaron, allí se señalan criterios y pautas a seguir. Identificaron algunos aspectos específicos, de la planeación y evaluación: a) planear clases dinámicas, b) tomar en cuenta los tres canales de aprendizaje, c) utilizar material concreto y d) realizar una evaluación permanente en donde se valore lo conceptual, procedimental y actitudinal; de la gestión del ambiente de clase: propiciar el respeto y la confianza permitiendo en todo momento que los alumnos se expresen, dialoguen, enseñen y aprendan entre sí; de la gestión didáctica: tener presentes los propósitos, los contenidos que se pretenden desarrollar y los aprendizajes esperados, para con ello atender las necesidades individuales de sus alumnos, respetando el trabajo individual y colectivo.

En la elaboración de su plan de mejora, los profesores pusieron en práctica su creatividad al diseñar secuencias didácticas y actividades innovadoras en un clima de ayuda mutua, disposición y respeto. Con 
ello, tuvieron la oportunidad de movilizar sus saberes previos. A este respecto, Pozo (1997) señaló que "una vez que una nueva estructura ha sido operada a su pensamiento, generalmente a través de conceptos adquiridos recientemente en la escuela, se expanden gradualmente entre los viejos conceptos" (p. 204).

Hubo una transformación de la visión de los profesores respecto a la evaluación de la práctica docente, lo que aporta elementos a una cultura de la evaluación del colectivo de profesores, pues allí reconocieron que en un plan de mejora se deben identificar con claridad las áreas de oportunidad, trazar objetivos y actividades que sean viables, pero sobre todo estar en una constante evaluación de la práctica para la mejora de la escuela. Una profesora comentó: "Algo que no podemos perder de vista en nuestras clases es manejar los tres aspectos de la evaluación, que son lo conceptual, lo procedimental y lo actitudinal". Además, los participantes señalaron que en ese proceso, el rol de quien esté a cargo de las funciones directivas deberá desarrollarse de tal forma que involucre a todo el personal para que cada profesor elabore su plan de mejora y se le dé un seguimiento a través de los CTE en una CoP.

\section{Análisis de los datos}

De los CTE como espacio de formación continua. La percepción que los profesores tuvieron de los CTE fue que, conformados en una CoP, les permitieron intercambiar experiencias que posibilitan cambios en su visión sobre la práctica docente. Esto es, al intercambiar experiencias entre iguales puedan alcanzar logros educativos significativos y, con ello, mejorar la apropiación de los conocimientos de su quehacer docente.

Señalaron que el intercambio de experiencias fue de mucho beneficio, pues a partir de ello tomaron acuerdos que aplicaron a sus propias clases, como lo comentó uno de ellos "compartir experiencias de la realidad que vivimos los docentes hoy en día en el aula nos permite aprender y compartir con los demás para favorecer una práctica docente cada vez más efectiva". Así reconocieron la importancia de la formación continua, resaltando que lo importante es que tenga repercusión en el aula y que se debe de hacer a la par de su práctica profesional a través de actividades innovadoras con "el propósito de mejorar el aprendizaje de los alumnos y el desarrollo de competencias" (E1PG2). Señalaron, además, que se debe estar en constante actualización para mejorar las prácticas y estar preparados para los retos que se presenten en las continuas reformas educativas, lo que contrasta con lo dicho por Imbernón (2006):

Los maestros y las maestras por cultura profesional no tienen la formación permanente como parte intrínseca de su profesión; o sea, un médico o un abogado, cuando acaba la carrera tiene muy claro que si quiere ganarse la vida tendrá que continuar estudiando y actualizándose. El maestro no tiene esta mentalidad, él acaba la formación inicial y piensa que el conocimiento le va a durar el tiempo (p. 5).

Contrario a lo planteado por Imbernón (2006), en el grupo de estudio los profesores tuvieron claridad sobre la importancia de su actualización, particularmente la que pueden desarrollar con su grupo de compañeros. Reconocieron que la formación continua es indispensable para el trabajo del profesor y que como parte de esa formación continua el trabajo colaborativo tiene gran importancia. De allí que se puede señalar que los profesores asumieron el compromiso de intercambiar experiencias en el CTE, en donde no se puede considerar la práctica del profesor como única, sino que se debe estar en constante reflexión considerando las ideas de otros profesores para transformar las propias a través de un trabajo colaborativo y, además, para fortalecer su formación profesional.

Desarrollo del trabajo colaborativo en CoP dentro del CTE en escuela de Educación Preescolar. En su participación en el proyecto, los profesores reflexionaron, hablaron y explicaron ante el colectivo la manera en que realizaban las prácticas evaluativas en su práctica docente y se percataron de que existe una diversidad de instrumentos que permiten ver con claridad cómo se apropian los alumnos de los diferentes saberes, abordándolo sobre dos aspectos: a) conformación de una cultura de evaluación y b) formación continua. 
Trabajo colaborativo en la conformación de una cultura de evaluación de la práctica docente. Los profesores reconocieron que las prácticas evaluativas que utilizaron en años anteriores no les permitían rescatar e identificar información suficiente para analizar la situación de sus alumnos, ya que se realizaba de una forma superflua y la información no se utilizaba con ningún fin que evidenciara las evaluaciones que realizaban. Indicaron que tenían dudas e inquietudes para aplicar las nuevas prácticas evaluativas propuestas por la SEP en la Reforma Integral de la Educación Básica.

Señalaron algunos aspectos que deben considerarse "de suma importancia" al realizar las evaluaciones, como: a) los conocimientos previos y b) orientar las actividades hacia el desarrollo de experiencias prácticas en las que apliquen lo aprendido. Así, un aspecto importante rescatado entre ellos fue la planeación de una situación didáctica, definida como un conjunto de actividades relacionadas entre sí que llevan a un mismo fin, coincidiendo con los planteamientos de la SEP (2011a).

Utilizar los Estándares de Evaluación del Desempeño Docente (Organización de Estados Iberoamericanos [OEI], 2008) con el fin de definir un poco más los aspectos que se deben tomar en cuenta dentro de la evaluación les facilitó identificar con más claridad los referentes para evaluar. Algunos de esos referentes eran desconocidos para los profesores, pero dentro del análisis realizado pudieron vincular acciones que efectuaban en la evaluación, las cuales buscaron transformar para aplicar en el aula.

Otro aspecto identificado en la reflexión sobre sus prácticas evaluativas fue que debían propiciar mejoras surgidas de un análisis de la práctica realizada con la sistematización de la informació: el uso de registros, listas de cotejo y las evaluaciones a través del análisis del desempeño de cada alumno. Una educadora comentó: "Debemos aprender sobre los tipos de evaluación, nosotras sólo utilizamos la observación, pero hay diferentes instrumentos, hojas de cotejo y tantas y tantas cosas que nos pueden apoyar". Sin embargo, aunque reconoció la importancia de la evaluación para tener más elementos en la toma de decisiones, se identificó que aún se requiere trabajar para transformar las prácticas evaluativas fomentando una cultura de evaluación en el contexto educativo, en donde entre todos los profesores conformen una visión no estática, sino que se va transformando conforme los participantes interactúan. Como planteó Torres (2006), esa cultura se conforma cuando en las interacciones sociales cotidianas los participantes cambian su actitud personal frente a la vida, lo que posibilita "la apertura a diversas perspectivas del mundo, su integración y acoplamiento al mismo y la inserción sin trauma a los diversos espacios sociales del mundo de la vida" (p. 92). Aspecto reconocido por los profesores, quienes consideraron que era indispensable generar esa cultura de la evaluación entre ellos.

De los CTE como espacio de formación continua. Los profesores reconocieron que el CTE es un espacio en el cual se tiene la oportunidad de interactuar y al hacerlo pudieron intercambiar experiencias que les permitió actualizarse en la práctica, en situaciones reales y contextualizado, el expresar cómo se trabaja tal o cual contenido y citar qué elementos conceptuales soportan el trabajo realizado. Una educadora mencionó que "no hay más que la práctica. Aquí vamos más a practicar que a ver teoría. Seguir preparándonos al ver que este instrumento me va a servir para esto, conocerlos y saber cuál es su función". Así reconocieron, por un lado, la importancia de la formación continua a la par de su práctica profesional, y por otro que para su actualización tienen la CoP, pues les permitió compartir experiencias y formas de trabajar contenidos específicos, así como herramientas de trabajo.

Aun cuando en el plazo inmediato el principal favorecido sea el docente, a mediano y largo plazo eso redundará en beneficio de sus alumnos y la función de la escuela. Al respecto, Álvarez y Romero (2007) expresaron que la profesionalización docente debe ser un ejercicio que vaya a la par de su trabajo en la institución, donde el profesor sea protagonista y su acción central sea el trabajo colaborativo a través del continuo cuestionamiento y la reflexión que le permita aterrizar en el aula lo compartido en el colectivo.

\section{Conclusiones}

Trabajar en los CTE en forma colaborativa en un espacio constituido como una CoP en la que los profesores pudieron analizar sus prácticas de evaluación desde su experiencia, pero apoyados con algunos elementos teóricos, les posibilitó identificar o reafirmar la importancia de analizar la evaluación que desarrollaban y sus concepciones a ese respecto, así como reconocer la riqueza de compartir esas 
experiencias y trazar líneas para su mejora. Sin embargo, todavía se requiere trabajar para que se construya una cultura de evaluación en el contexto educativo específico, en la que en el centro se encuentre la revisión de sus prácticas, la transformación que requieren y se incorporen a las prácticas en un proceso dialéctico.

El trabajo colaborativo desarrollado en los CTE los fortaleció como espacios para la formación continua. Los participantes señalaron que tuvieron oportunidad de intercambiar experiencias enriquecedoras y elementos teóricos -como herramientas conceptuales- para entender mejor la práctica cotidiana y no tratar de acotar la realidad a la teoría.

Los profesores construyeron una visión de los CTE como una plataforma para que lo que allí se trabaje, a la par del desarrollo de su práctica, repercuta directamente en lo que en ella realizan. Así, aunque la participación sea establecida normativamente, se mostraron de acuerdo en que aunque participen en cursos de actualización, resulta más enriquecedor compartir experiencias, recursos didácticos y materiales para utilizarlos en su grupo. Por todo ello, puede plantearse que se generaron ciertas bases para que los profesores continúen trabajando en esa dinámica para que poco a poco pueda constituirse en una forma dinámica de desarrollar los CTE y que el análisis de las prácticas de evaluación sea un de los aspectos centrales a abordar en ellos.

\section{Referencias}

Álvarez, V. y Romero, S. (2007). Formación basada en competencias para profesionales de la orientación. Educación XXI, 10, 15-37. Recuperado de

http://revistas.uned.es/index.php/educacionXX1/article/view/295

Casanova, M. A. (2004). Evaluación y calidad de centros educativos. Madrid: La Muralla.

Ezpeleta, J. (1990). El Consejo Técnico: eficacia pedagógica y estructura de poder en la escuela primaria mexicana. Revista Latinoamericana de Estudios Educativos, 20(4), 1333. Recuperado de

www.cee.edu.mx/revista/r1981 1990/r texto/t 19904 02.pdf

Fierro, C. y Rojo, S. (1994). El consejo técnico, Un encuentro de maestros. Recuperado de http://es.scribd.com/doc/28993719/El-ct-Un-Encuentro-de-Maestros-eBook

González, R. M., Castañeda, A., Torres, M., Banda, R., Vargas, R. y Ruíz, F. (2013). Colaboración en comunidad de práctica para el desarrollo profesional del profesor. Revista de Medios y Educación Pixel-Bit, 42, 103-113. Recuperado de http://acdc.sav.us.es/ojs/index.php/pixelbit/article/view/466

González, R. M., Zamarripa, S., Cardoso, A., Martínez, R. y Sánchez, A. (2009). Análisis de la práctica pedagógica en video: experiencia en comunidad de práctica de escuela primaria. En J. S. Sánchez y A. Rangel Ruiz de la Peña, Maestros y formación en comunidades de práctica. México: Universidad Pedagógica Nacional.

Organización de Estados Iberoamericanos. (2008). Estándares de evaluación docente. México: Autor.

Imbernón, F. (2006). Actualidad y nuevos retos en la formación permanente. Revista Electrónica de Investigación Educativa, 8(2), 1-12. Recuperado de http://redie.uabc.mx/redie/article/view/151

González, K. (2009). Propuesta estratégica y metodológica para la gestión en el trabajo colaborativo. Educación, 2(33), 95-107. Recuperado de http://revistas.ucr.ac.cr/index.php/educacion/article/view/507

López, Y. (2005). La Universidad Pedagógica Nacional. Una imagen mitificada donde el relato actuado se hace creíble. Tesis Doctoral. México: Universidad Pedagógica Nacional. 
Lucero, M. M. (2009). Entre el trabajo colaborativo y el aprendizaje colaborativo. Revista Iberoamericana de Educación. Recuperado de http://www.rieoei.org/deloslectores/528Lucero.PDF

Marín, R., Guzmán, I. y Castro, G. (2012). Diseño y validación de un instrumento para la evaluación de competencias en preescolar. Revista Electrónica de Investigación Educativa, 14(1), 182-202. Recuperado de http://redie.uabc.mx/redie/article/view/308

Messina, G. (2002). Formación en movimiento: una propuesta a distancia. Memoria de la Reunión Regional para Evaluar las Estrategias y Programas para el aprendizaje de jóvenes y adultos en América Latina y el Caribe. Recuperado de

http://bibliotecadigital.conevyt.org.mx/colecciones/documentos/reunion/ponencias/forma docente/pon encia graciela mesina crefal septiembre.pdf

Moliner, L., Castellón, J. y Loren, C. (2010). La Formación Continua como proceso clave en la profesionalización docente: buenas prácticas en Chile. Revista Latinoamericana de Educación Inclusiva, 4(1), 25-44. Recuperado de http://www.rinace.net/rlei/numeros/vol4-num1/art1.pdf

Orler, P. y Abendaño, S. (2009). Las reuniones colegiadas estrategia para conocer y orientar a los alumnos. Revista Novedades Educativas, 21(218), 1-5.

Pozo, J. I. (1997). Teorías Cognitivas del aprendizaje. España: Morata.

Scagnoli, N. I. (2005). Estrategias para motivar el aprendizaje colaborativo en cursos a distancia. Recuperado de https://www.ideals.illinois.edu/handle/2142/10681

Secretaría de Educación Pública. (1982). Acuerdo 96, Acuerdo que establece la organización y funcionamiento de las escuelas primarias. México: Autor.

Secretaría de Educación Pública y Sindicato Nacional de Trabajadores de la Educación. (2011a). Acuerdo 592 por el que se establece la articulación de la Educación Básica. México: Autor.

Secretaría de Educación Pública. (2011b). Plan de Estudios 2011. Educación Básica. México: Autor.

Tallaferro, D. (2006). La formación para la práctica reflexiva en las prácticas profesionales docentes. Educare , 10(33), 269-273. Recuperado de http://www.redalyc.org/articulo.oa?id=35603309

Torres, A. (2006). Subjetividad y sujeto: perspectivas para abordar lo social y lo educativo. Revista Colombiana de Educación, 50, 87-103

Wenger, É. (2002). Comunidades de práctica: aprendizaje, significado e identidad. España: Paidós. 